\title{
RESEARCH
}

Open Access

\section{Cardiac structural changes after transcatheter aortic valve replacement: systematic review and meta-analysis of cardiovascular magnetic resonance studies}

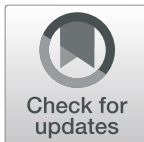

Ghazaleh Mehdipoor ${ }^{1}$, Shmuel Chen ${ }^{1,2}$, Saurav Chatterjee ${ }^{3}$, Pooya Torkian ${ }^{4}$, Ori Ben-Yehuda ${ }^{1,2}$, Martin B. Leon ${ }^{1,2}$, Gregg W. Stone $e^{1,5}$ and Martin R. Prince ${ }^{6,7^{*}}$

\begin{abstract}
Background: Transcatheter aortic valve replacement (TAVR) is increasingly used to treat patients with severe aortic stenosis (AS). Cardiovascular magnetic resonance imaging (CMR) provides reliable and reproducible estimates for assessment of cardiac structure and function after TAVR. The goal of this study was to conduct a systematic review and meta-analysis of the literature to assess left ventricular (LV) volumes, mass and function by CMR after TAVR.

Methods: Using Meta-analysis of Observational Studies in Epidemiology (MOOSE) guidelines, we searched PubMed and Embase for studies reporting CMR findings before and at least 1 month after TAVR. Main factors of interest were LV end-diastolic volume index (LVEDVi), LV end-systolic volume index (LVESVi), LV mass index (LVMi), and left ventricular ejection fraction (LVEF). Standardized mean differences (SMD) were pooled by random effects metaanalytic techniques.
\end{abstract}

Results: Of 453 screened publications, 10 studies (published between 2012 and 2018) were included. A total of 305 patients completed pre- and post-TAVR follow-up CMR (mean age range 78.6-85.0 years, follow-up range 6-15 months). Random effects analysis showed TAVR resulted in reduced LVEDVi (SMD: $-0.25,95 \%$ Cl: -0.43 to -0.07 , $P=0.006)$, LVESVi (SMD: $-0.24,95 \% \mathrm{Cl}:-0.44$ to $-0.05, P=0.01)$, LVMi (SMD: $-0.82,95 \% \mathrm{Cl}:-1.0$ to $-0.63, P<0.001$ ) and increased LVEF (SMD: $22,95 \% \mathrm{Cl}: 6$ to $38 \%, P=0.006)$. Heterogeneity across studies was low $\left(1^{2}: 0 \%\right.$, $P_{\text {heterogeneity }}>0.05$ for all). The median reduction was $4 \mathrm{ml} / \mathrm{m}^{2}$ (IQR: 3.1 to 8.2 ) for LVEDVi, $5 \mathrm{ml} / \mathrm{m}^{2}$ (IQR: 3.0 to 6.0) for LVESVi, and $15.1 \mathrm{~g} / \mathrm{m}^{2}$ (IQR: 11.8 to 18.3) for LVMi. The median increase for LVEF was 3.4\% (IQR 1.0 to $4.6 \%$ ).

Conclusions: CMR demonstrates reverse LV remodeling occurrs within 6-15 months after TAVR, with reductions in LVEDVi, LVESVi and LVMi, and increased LVEF.

Keywords: Transcatheter aortic valve replacement (TAVR), Transcatheter aortic valve implantation (TAVI), Aortic stenosis, Magnetic resonance imaging (MRI), Cardiac structure

\footnotetext{
* Correspondence: map2008@med.cornell.edu

${ }^{6}$ Department of Radiology, Weill Cornell Medical College\& New York Presbyterian Hospital, 416 East 55th Street, New York, NY 10022, USA

7Department of Radiology, Columbia University College of Physicians and Surgeons, New York, NY, USA

Full list of author information is available at the end of the article
}

(c) The Author(s). 2020 Open Access This article is licensed under a Creative Commons Attribution 4.0 International License, which permits use, sharing, adaptation, distribution and reproduction in any medium or format, as long as you give appropriate credit to the original author(s) and the source, provide a link to the Creative Commons licence, and indicate if changes were made. The images or other third party material in this article are included in the article's Creative Commons licence, unless indicated otherwise in a credit line to the material. If material is not included in the article's Creative Commons licence and your intended use is not permitted by statutory regulation or exceeds the permitted use, you will need to obtain permission directly from the copyright holder. To view a copy of this licence, visit http://creativecommons.org/licenses/by/4.0/ The Creative Commons Public Domain Dedication waiver (http://creativecommons.org/publicdomain/zero/1.0/) applies to the data made available in this article, unless otherwise stated in a credit line to the data. 


\section{Introduction}

Aortic stenosis (AS) is the most common valvular heart disease in the developed world, with increased prevalence in the aging population [1]. AS is associated with cardiac remodeling due to the pressure overload including a compensatory gradual left ventricular (LV) hypertrophy, impaired LV diastolic filling, which may ultimately lead to compromised LV function in some patients [2-5]. Surgical aortic valve replacement has been the standard of care for treatment of AS for many years; however, during the past decade, transcatheter aortic valve replacement (TAVR) is increasingly used to treat patients with severe AS, especially when there are comorbidities that increase the surgical risk [6].

Monitoring cardiac structure and function post-TAVR is usually performed by transthoracic echocardiography (TTE), which is widely available and portable. However, TTE is prone to inter-observer variability, and limited in several patient subgroups such as obese patients or patients with hyper-inflated lungs [7].

Cardiovascular magnetic resonance imaging (CMR) provides more reliable estimates for assessment of cardiac structure and function and is the non-invasive gold standard imaging modality for these purposes [8]. However, prior CMR studies on cardiac remodeling post-TAVR have been small, with variability in the results across some studies [911]. These limitations have led to uncertainty about the presence and magnitude of changes in ventricular structure and function after TAVR as assessed by CMR [12]. Therefore, we conducted a systematic review and meta-analysis to evaluate the changes in LV volumes, mass, and function assessed by CMR in patients undergoing TAVR.

\section{Methods}

\section{Data source}

We searched PubMed and Embase for original studies that reported CMR findings before and at least 1 month postTAVR, from January 1, 2000 to August 23, 2018, using a combination of Medical Subject Heading (MeSH) terms and keywords, in accordance with Meta-analysis of Observational Studies in Epidemiology (MOOSE) guidelines (Supplementary Table 1) [13]. The MOOSE guidelines have been developed to help with standardized data design, data abstraction, meta-analysis, and reporting of observational studies. The guidelines also address some of the challenges of the observational studies such as the differences in study design, inherent biases (e.g. selection bias), and confounding factors such as varying disease severity [14].

\section{Study selection}

All titles and abstracts of the retrieved studies were screened and where needed, the full manuscript text was reviewed for inclusion. We also searched the reference lists of included studies to identify additional potentially relevant studies. We included all cohort studies and case series that reported CMR findings before and at least 1 month after TAVR. The choice of the time interval was by authors' consensus, so that there would be sufficient time for acute changes such as myocardial edema to resolve, and for reverse remodeling to occur $[12,15]$. No language restriction nor sample size restriction were applied in selecting the articles. We excluded non-human studies, as well as ex-vivo heart assessments. Where the data from the same center were presented in multiple publications, we only included the results of the most comprehensive report from that center to avoid duplicate data.

\section{Data extraction and study outcomes}

For each study, the data screening and abstraction was performed by 2 independent investigators (GM, and PT). All discrepancies were resolved by consensus. In addition, the investigators of primary studies were contacted for clarifications or additional data, where relevant data were missing. Main factors of interest were LV end-diastolic volume index (LVEDVi), LV end-systolic volume index (LVESVi), LV mass index (LVMi), and left ventricular ejection fraction (LVEF).

\section{Quality assessment}

Assessing the quality of included studies, especially the appropriate reporting of the methodological details is critical for interpretation of the meta-analysis results. We assessed the methodological quality of the included full-text studies using the Newcastle-Ottawa scale as recommended by Cochrane Collaboration [16]. The Newcastle-Ottawa scale scores each study to a maximum of 9 , on 3 main areas of the selection of the study groups, comparability of the groups, and the ascertainment of the outcome of interest [17].

\section{Statistical analysis}

Standardized mean differences (SMD) from the included studies were pooled by conventional random effects meta-analytic techniques, to account for the variations across the studies. Random effects meta-analysis assumes that the estimated effects in different studies are not identical [18]. The degree of heterogeneity across studies was assessed by the I-squared $\left(\mathrm{I}^{2}\right)$. The $\mathrm{I}^{2}<25 \%$ was considered as low heterogeneity, and $\mathrm{I}^{2}>75 \%$ as high heterogeneity [19]. We made a priori plans not to pool the data for variables that were reported by fewer than 3 studies. To account for possible differences in studies over time, we also performed a random effects meta-regression analysis, with year of publication and duration of follow-up as covariates, to verify the robustness of the main results. We used funnels plot and the Harbord's regression modification of Egger's test to assess potential publication bias for the small study effect 
[20]. A two-sided $P$-value of $<0.05$ was considered as significant. We used STATA software, version 12.0 (StataCorp, College Station, Texas, USA) for all analyses.

\section{Results}

A total of 453 publications were identified through our search of PubMed (150 records), and Embase (303 records). Following the screening of the titles and abstracts, 36 articles were selected for full-text review. No additional records were found through hand search of the reference lists of the retrieved articles. Finally, after excluding articles not meeting inclusion criteria or duplicate studies [21-24], 10 studies with a total of 12 study cohorts were included for meta-analysis (Fig. 1)
[9-11, 25-31]. The studies were published between 2012 and 2018, and included a total of 305 patients who completed both pre- and post TAVR CMR. For all studies, follow-up CMR was performed at least 6-month post-TAVR, and all studies were performed at $1.5 \mathrm{~T}$ using a standard CMR protocol using the balanced steady-state free precession pulse sequence (Table 1).

\section{Left ventricular changes}

CMR at follow-up showed a significant reduction in LVEDVi (9 cohorts; 242 patients; SMD: -0.25, 95\% CI: 0.43 to $-0.07, P=0.006)$. No evidence of heterogeneity was observed between the included studies $\left(\mathrm{I}^{2}: 0 \%, \mathrm{P}_{\text {hetero- }}\right.$ geneity $=0.97)$ (Fig. 2a). The median study-level average

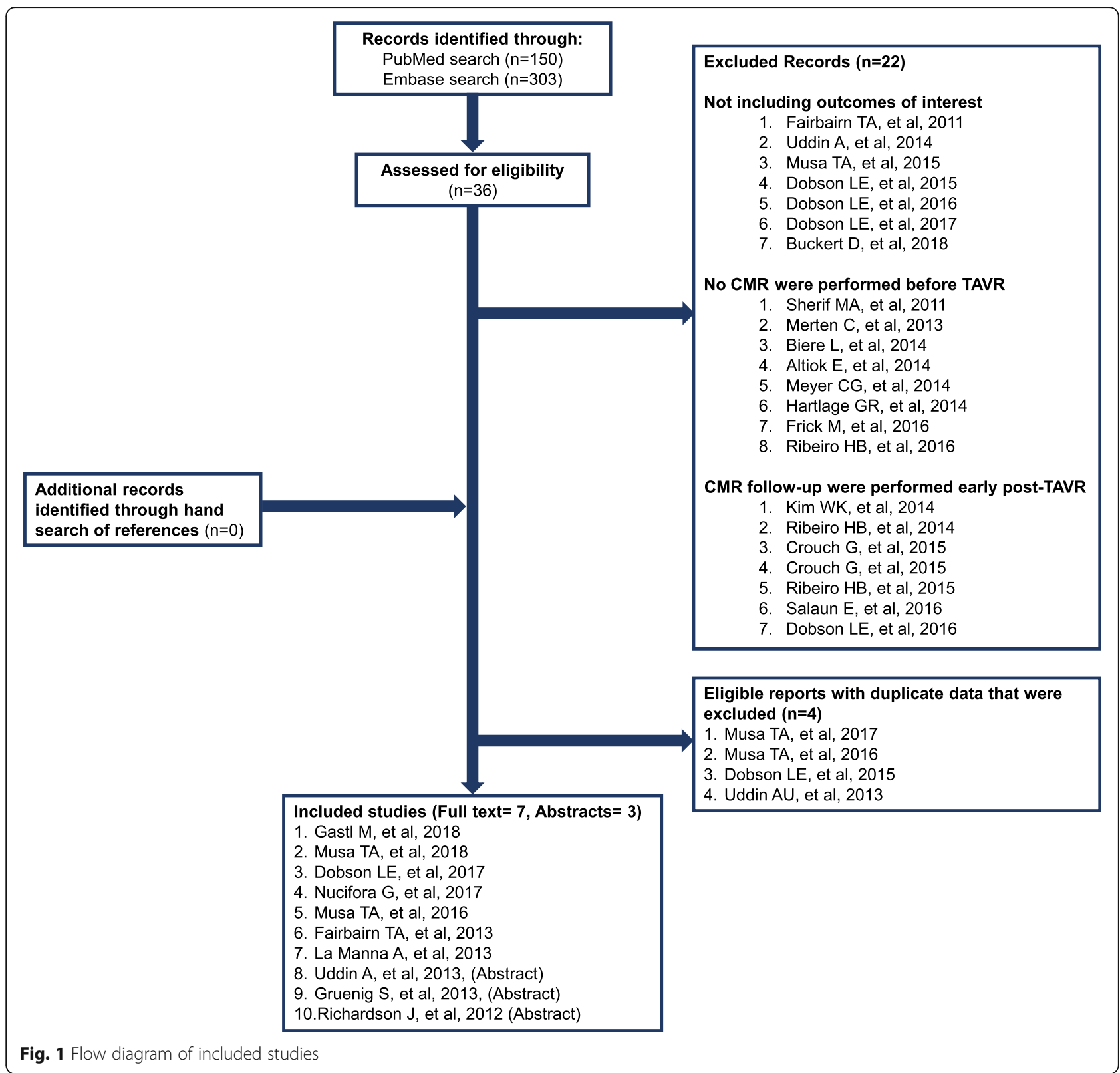




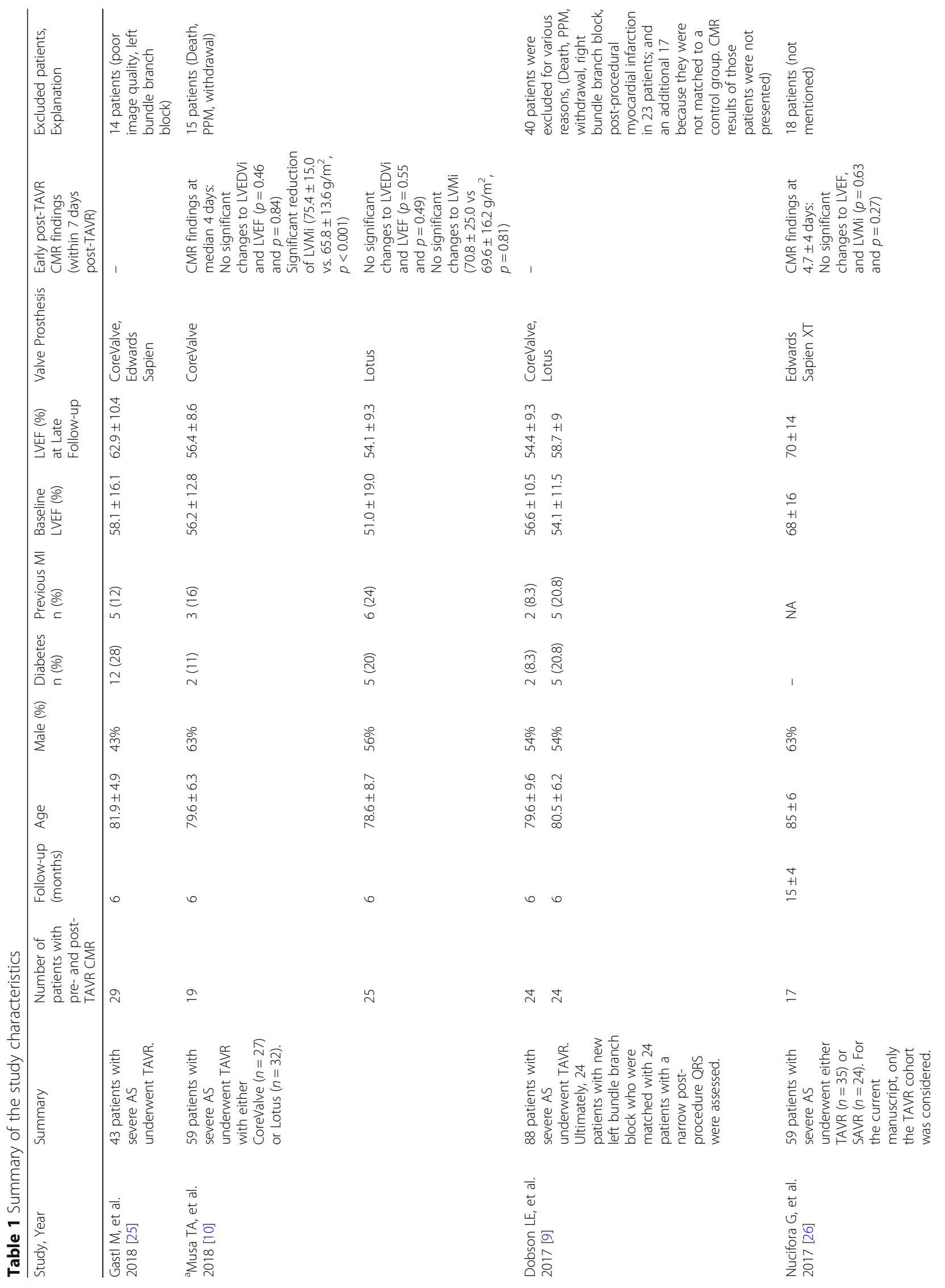




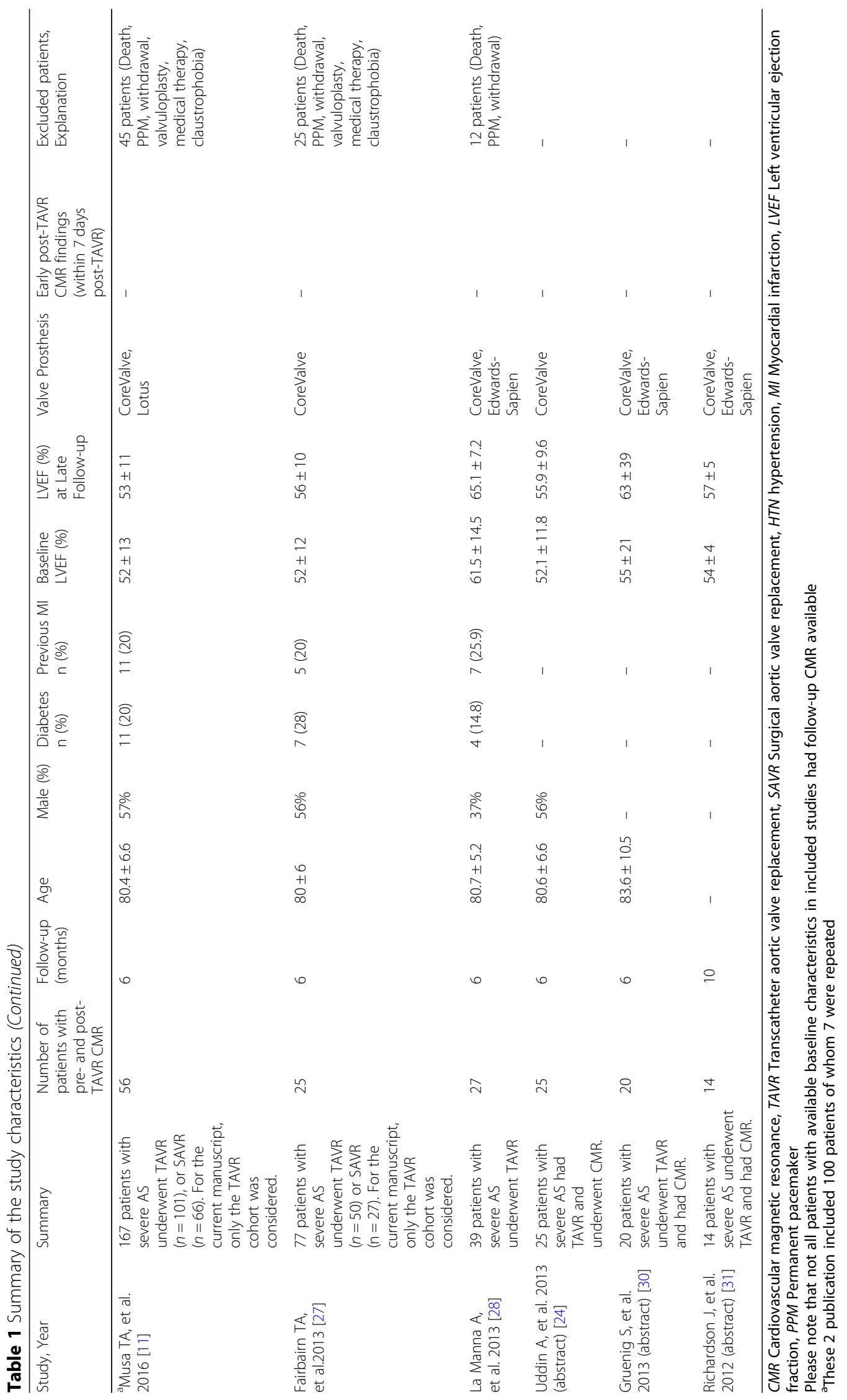




\section{a. LVEDVi}

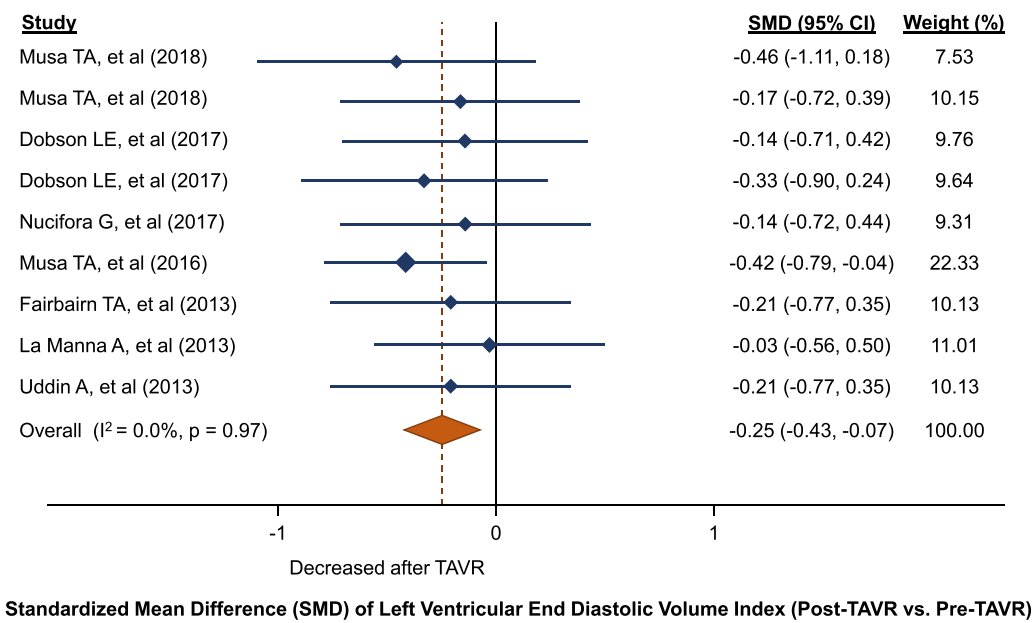

Fig. 2 Left ventricular (LV) changes, standardized mean difference (SMD) post-TAVR Vs. pre-TAVR for: a. LVEDVi, b. LVESVi, c. LVMi, d. LVEF. LVEDVi, left ventricular end-diastolic volume index; LVEF, left ventricular ejection fraction; LVESVI, left ventricular end-systolic volume index, LVMi, left ventricular mass index; TAVR, transcatheter aortic valve replacement

reduction in LVEDVi was $4.0 \mathrm{ml} / \mathrm{m}^{2}$ (interquartile range [IQR]: 3.1 to 8.2 ).

LVESVi was also reduced after TAVR (7 cohorts; 198 patients; SMD: -0.24 , 95\% CI: -0.44 to $-0.05, P=0.01)$ with no evidence of heterogeneity $\left(\mathrm{I}^{2}: 0 \%, \mathrm{P}_{\text {heterogeneity }}=\right.$ 0.96) (Fig. 2b). The median study-level average reduction for LVESVi was $5.0 \mathrm{ml} / \mathrm{m}^{2}$ (IQR: 3.0 to 6.0 ).

LVMi was significantly reduced after TAVR ( 9 cohorts; 246 patients; SMD: $-0.82,95 \%$ CI: -1.0 to $-0.63, P<$ 0.001 ). Across the included studies, no evidence of heterogeneity was observed $\left(\mathrm{I}^{2}: 0 \%, \mathrm{P}_{\text {heterogeneity }}=0.58\right)$ (Fig. 2c). The median average reduction for LVMi was $15.1 \mathrm{~g} / \mathrm{m}^{2}$ (IQR: 11.8 to 18.3 ).

LVEF was significantly increased after TAVR (12 cohorts; 305 patients; SMD: 22, 95\% CI: 6 to $38 \%, P=0.006$ ). Among the included studies, there was no evidence of heterogeneity $\left(I^{2}: 0 \%, P_{\text {heterogeneity }}=0.87\right)$ (Fig. $\left.2 d\right)$. Across the studies, the median average LVEF at baseline was 54.6\% (IQR: 52.0 to $58.1 \%$ ) and the median average increase was $3.4 \%$ (IQR 1.0 to $4.6 \%$ ).

\section{b. LVESVi}

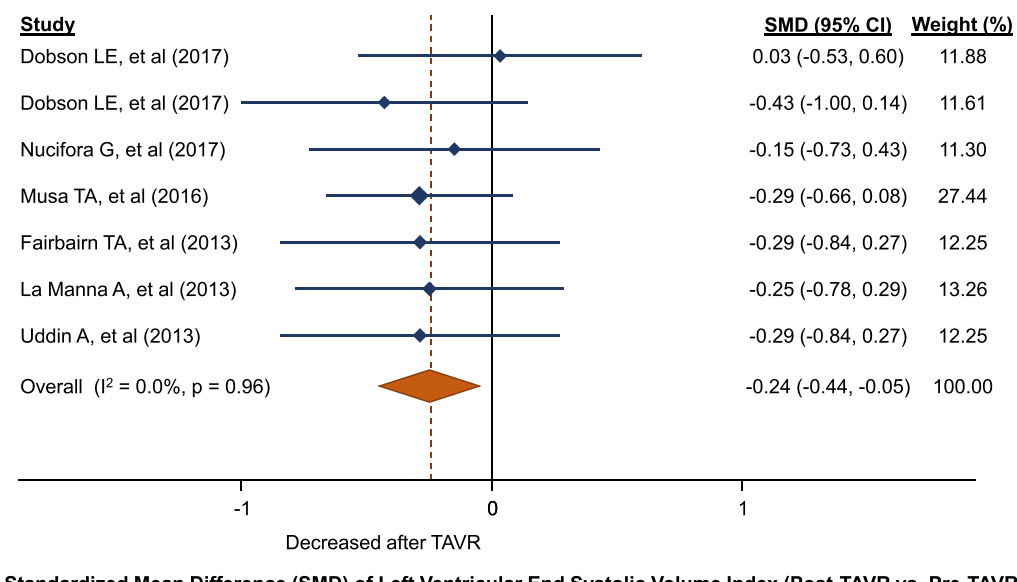

Fig. 2 Left ventricular (LV) changes, standardized mean difference (SMD) post-TAVR vs. pre-TAVR for: a. LVEDVi, b. LVESVi, c. LVMi, d. LVEF. LVEDVi, left ventricular end-diastolic volume index; LVEF, left ventricular ejection fraction; LVESVI, left ventricular end-systolic volume index, LVMi, left ventricular mass index; TAVR, transcatheter aortic valve replacement 


\section{c. LVMi}

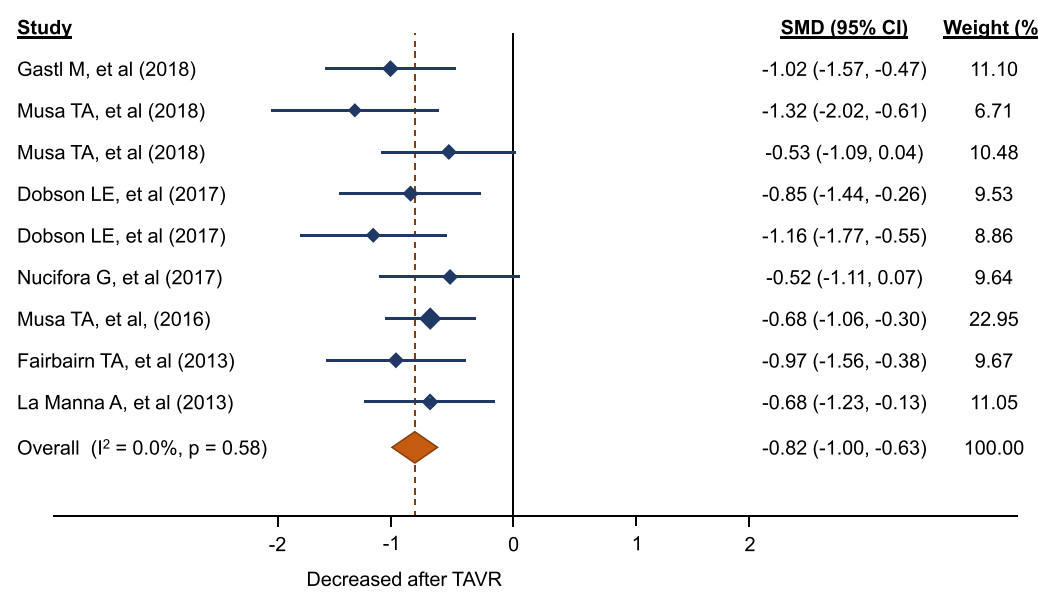

Standardized Mean Difference (SMD) of Left Ventricular Mass Index (Post-TAVR vs. Pre-TAVR)

Fig. 2 Left ventricular (LV) changes, standardized mean difference (SMD) post-TAVR vs. pre-TAVR for: a. LVEDVi, b. LVESVi, c. LVMi, d. LVEF. LVEDVi, left ventricular end-diastolic volume index; LVEF, left ventricular ejection fraction; LVESVI, left ventricular end-systolic volume index, LVMi, left ventricular mass index; TAVR, transcatheter aortic valve replacement

\section{Sensitivity analysis}

In a post-hoc sensitivity analysis, we repeated the analyses for LVEDVi, LVESVi, LVMi, and LVEF after excluding 3 studies that were presented as abstracts. Although the point estimates had modest changes, the results were consistent, suggestive of significant reduction in LVEDVi, LVESVi, and LVMi, and improvement in LVEF (Table 2, Supplementary Figure 1).

\section{Risk of bias assessment}

The quality of included studies was assessed using the Newcastle-Ottawa Scale. The total score varied between $6 / 9$ and $8 / 9$. The most frequent limitations were the lack of representation of the exposed cohort, and incomplete follow-up (Supplementary Table 2).

\section{d. LVEF}

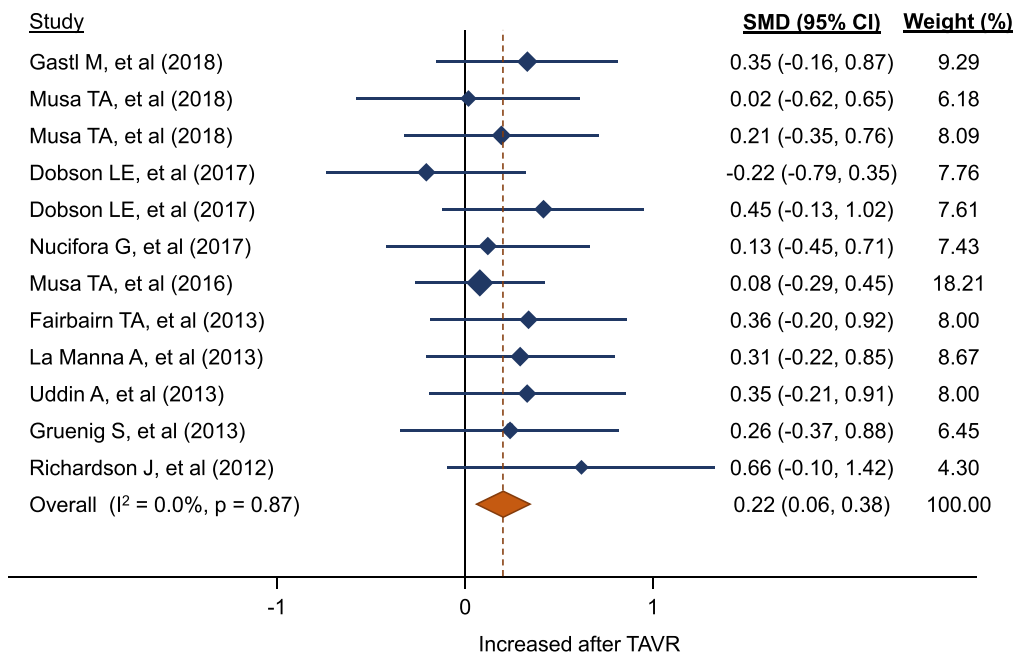

Standardized Mean Difference (SMD) of Left Ventricular Ejection Fraction (Post-TAVR vs. Pre-TAVR)

Fig. 2 Left ventricular (LV) changes, standardized mean difference (SMD) post-TAVR vs. pre-TAVR for: a. LVEDVi, b. LVESVi, c. LVMi, d. LVEF. LVEDVi, left ventricular end-diastolic volume index; LVEF, left ventricular ejection fraction; LVESVI, left ventricular end-systolic volume index, LVMi, left ventricular mass index; TAVR, transcatheter aortic valve replacement 


\section{Publication bias}

No publication bias was apparent from visual inspection of the funnel plots for LVEDVi, LVESVi, LVEF, and LVMi (Supplementary Figure 2) and this was confirmed quantitatively with the Harbord's regression modification of Egger's test $(P>0.25$ for all).

\section{Meta-regression}

Meta-regression analysis did not show a significant association between either the duration of follow-up postTAVR or the year of study publication and any of LVEDVi, LVESVi, LVEF and LVMi measurements $(P>$ 0.25 for all) (Supplementary Table 3).

\section{Discussion}

This systematic review and meta-analysis indicates that among patients undergoing TAVR who completed the follow-up CMR 6-15 months after the procedure, there was evidence for reverse LV remodeling. The decrease observed in LVEDVi and LVESVi likely reflects improved preload and afterload. Further, the significant reduction in LVMi toward normal values, along with the increased LVEF are consistent with gradual reverse LV remodeling after TAVR. This study adds to the existing literature by providing comprehensive estimates of association using the existing published data from prior individual studies on the topic [9-11, 25-31].

The prognostic impact of maladaptive cardiac remodeling, as well as reverse remodeling are well recognized in several cardiac conditions [32-34]. Interestingly, prior studies have indicated that irreversibility of changes or lack of improvement in LVEDVi, and LVMi post-TAVR are associated with worse outcomes [35]. Whereas an improvement in these indices is associated with better survival [35-37]. Despite the value of those studies, such investigations were based on TTE, which is subject to image acquisition limitations, as well as inter-observer and intra-observer variability [38]. To the best of our knowledge, this is the first systematic review of the literature to assess the cardiac structural changes by CMR, post-TAVR. These results are complementary to the

Table 2 Left ventricular changes, degree of heterogeneity $\left(1^{2}\right)$ across the included studies after excluding 3 studies that were presented as abstracts

\begin{tabular}{lllll}
\hline & SMD $(95 \% \mathrm{Cl})$ & $P$-value & $\mathrm{l}^{2}$ & P-heterogeneity \\
\hline LVEDVi & $0.23(0.03-0.44)$ & 0.02 & $0.0 \%$ & 0.91 \\
LVESVi & $0.25(0.07-0.43)$ & 0.007 & $0.0 \%$ & 0.94 \\
LVMi & $0.80(0.62-0.98)$ & $<0.001$ & $0.0 \%$ & 0.52 \\
LVEF & $18 \%(1-35 \%)$ & 0.04 & $0.0 \%$ & 0.82 \\
\hline
\end{tabular}

SMD Standardized mean difference, LVEDVi Left ventricular end diastolic volume index, LVESVi Left ventricular end systolic volume index, LVMi Left ventricular mass index, LVEF left ventricular ejection fraction existing TTE findings and provide additional evidence of cardiac reverse remodeling post-TAVR.

Our findings based on pooled results from CMR studies are consistent with the previous TTE findings for LVMi. TTE data from PARTNER trial have shown continuous regression of LVMi $\left(\mathrm{g} / \mathrm{m}^{2}\right)$ over time (from $155.6 \pm 40.6$ at baseline, to $148.6 \pm 38.3$ at 30 -day followup, to $140.4 \pm 38.1$ at 6 -month follow-up, to $135.7 \pm 37.9$ at 1 -year follow-up, and to $124.7 \pm 35.6$ at 2 -year followup) [37]. Further, TTE data from the PARTNER trial have shown improvement in LVEF (\%) after TAVR $(52.6 \% \pm 13.4$ at baseline, to $56.0 \% \pm 11.2$ at 30 -day follow-up, and to $56.6 \% \pm 10.4$ at 1-year follow-up) [37].

\section{Limitations}

Our study has several limitations. First, it is possible that some reports were not retrieved through our search strategy or were not indexed in PubMed. Second, it is clinically relevant to understand the pattern of reverse remodeling in certain patient subgroups, including those with concentric hypertrophy without LV dilation. In addition, mitral regurgitation is common in patients undergoing TAVR, and presence and severity of mitral regurgitation may also influence the remodeling [39]. However, detailed clinical and CMR data across the studies were not available to address this issue. Third, data regarding the valve type, and its potential effect on the cardiac remodeling was not available across the included studies. Fourth, the vast majority of included studies assessed the CMR characteristics 6 months post-TAVR. Additional studies are required to report post-TAVR CMR features in other time intervals. Fifth, there were a few (7/ 100) overlapping patients among 2 of the included studies. However, given the total number of patients in the pooled sample $(N=305)$, it is unlikely that such a modest overlap substantively impacted the results. Sixth, across the included studies, several patients were excluded from follow-up CMR post-TAVR due to permanent pacemaker implantation, death, or claustrophobia. It is possible that the patients who died or who had pacemakers have a different remodeling response to TAVR. This hypothesis is supported by subgroup analyses of prior relatively small TTE studies in patients with pacemakers, post-TAVR [40]. With the availability of CMR compatible pacemakers, as well as promising results of a recent investigation about the relative safety of CMR even in patients with traditional pacemaker devices [41], it is possible that future CMR studies further assess these subgroups.

\section{Conclusions}

In conclusion, CMR demonstrates reverse LV remodeling within 6-15 months after TAVR, with reductions in LVEDVi, LVESVi and LVMi, and improved LVEF. Better understanding of the reverse remodeling process among 
patients with different comorbidities, and the prognostic utility of these findings warrant further investigation.

\section{Supplementary information}

Supplementary information accompanies this paper at https://doi.org/10. 1186/s12968-020-00629-9.

Additional file 1. Supplementary Table S1. Search strategy.

Additional file 2. Supplementary Table S2. Quality assessment of included studies using the Newcastle-Ottawa Scale.

Additional file 3. Supplementary Table S3. Meta regression for year of study publication and follow-up months.

Additional file 4. Supplementary Figure S1. Left ventricular changes after excluding 3 abstracts, standardized mean difference (SMD) for: a LVEDVi, b. LVESVi, c. LVMi, d. LVEF.

Additional file 5. Supplementary Figure S2. Funnel plots for potential publication bias showing symmetric distribution for: a. LVEDVi, b. LVESVi, c. LVMi, d. LVEF.

\section{Abbreviations}

AS: Aortic stenosis; Cl: Confidence interval; CMR: Cardiovascular magnetic resonance; EDVi: End-diastolic volume index; EF: Ejection fraction; ESVi: End systolic volume index; HTN: Hypertension; LV: Left ventricle/left ventricular; LVMi: LV mass index; MeSH: Medical subject heading; MOOSE: Meta-analysis of Observational Studies in Epidemiology; SAVR: Surgical aortic valve replacement; SMD: Standardized mean differences; TAVR: Transcatheter aortic valve replacement; TTE: Transthoracic echocardiography

\section{Acknowledgements}

We would like to acknowledge Dr. John P. Greenwood for providing additional information for 2 of the included studies.

\section{Authors' contributions}

GM, SC, SC, PT, OB, MBL, GWS, MRP contributed to the conception, design, analysis, interpretation of data, drafting and revising the manuscript. GM, PT also contributed to data extraction. All authors read and approved the final manuscript.

\section{Funding}

None.

\section{Availability of data and materials}

The datasets used and analyzed during the current study are available from the corresponding author on reasonable request.

\section{Ethics approval and consent to participate}

Not applicable.

\section{Consent for publication}

Not applicable.

\section{Competing interests}

GM, SC, SC, PT, OB have no relevant Disclosures. Dr. Leon reports grants and other from Edwards Lifesciences, during the conduct of the study; grants and personal fees from Medtronic, grants and personal fees from Boston Scientific, personal fees from Gore Medical, personal fees from Meril Lifescience, grants and personal fees from Abbott, outside the submitted work. Dr. Stone is consultant to Gore. Dr. Prince has an unrelated patent agreement with GE Healthcare.

\section{Author details}

${ }^{1}$ Cardiovascular Research Foundation, New York, NY, USA. ${ }^{2}$ Department of Cardiology, Columbia University College of Physicians and Surgeons, New York, NY, USA. ${ }^{3}$ Hoffman Heart Institute, Saint Francis Hospital of the University of Connecticut, Hartford, CT, USA. ${ }^{4}$ Shahid Beheshti University of Medical Sciences, Tehran, Iran. ${ }^{5}$ The Zena and Michael A. Wiener Cardiovascular Institute, Icahn School of Medicine at Mount Sinai, New York,
NY, USA. ${ }^{6}$ Department of Radiology, Weill Cornell Medical College\& New York Presbyterian Hospital, 416 East 55th Street, New York, NY 10022, USA. ${ }^{7}$ Department of Radiology, Columbia University College of Physicians and Surgeons, New York, NY, USA.

Received: 1 November 2019 Accepted: 21 April 2020

Published online: 01 June 2020

\section{References}

1. Thaden JJ, Nkomo VT, Enriquez-Sarano M. The global burden of aortic stenosis. Prog Cardiovasc Dis. 2014;56(6):565-71.

2. Nkomo VT, Gardin JM, Skelton TN, Gottdiener JS, Scott CG, Enriquez-Sarano M. Burden of valvular heart diseases: a population-based study. Lancet (London, England). 2006;368(9540):1005-11.

3. Rassi AN, Pibarot P, Elmariah S. Left ventricular remodelling in aortic stenosis. Can J Cardiol. 2014;30(9):1004-11.

4. Rader F, Sachdev E, Arsanjani R, Siegel RJ. Left ventricular hypertrophy in valvular aortic stenosis: mechanisms and clinical implications. Am J Med 2015;128(4):344-52.

5. Yarbrough WM, Mukherjee R, Ikonomidis JS, Zile MR, Spinale FG. Myocardial remodeling with aortic stenosis and after aortic valve replacement: mechanisms and future prognostic implications. J Thorac Cardiovasc Surg. 2012;143(3):656-64.

6. Leon MB, Smith CR, Mack MJ, Makkar RR, Svensson LG, Kodali SK, et al. Transcatheter or surgical aortic-valve replacement in intermediate-risk patients. N Engl J Med. 2016;374(17):1609-20.

7. Malik SB, Chen N, Parker RA 3rd, Hsu JY. Transthoracic echocardiography: pitfalls and limitations as delineated at cardiac CT and MR imaging. Radiographics. 2017;37(2):383-406.

8. Lopez-Mattei JC, Shah DJ. When to consider cardiovascular magnetic resonance in patients undergoing transcatheter aortic valve replacement? Curr Opin Cardiol. 2013;28(5):505-11.

9. Dobson LE, Musa TA, Uddin A, Fairbairn TA, Bebb OJ, Swoboda PP, et al. The impact of trans-catheter aortic valve replacement induced left-bundle branch block on cardiac reverse remodeling. J Cardiovasc Magn Reson. 2017;19(1):22

10. Musa TA, Uddin A, Dobson LE, Swoboda PP, Garg P, Foley JRJ, et al. Cardiovascular magnetic resonance assessment of 1st generation CoreValve and 2nd generation lotus valves. J Interv Cardiol. 2018;31(3):391-9.

11. Musa TA, Uddin A, Fairbairn TA, Dobson LE, Steadman CD, Kidambi A, et al. Right ventricular function following surgical aortic valve replacement and transcatheter aortic valve implantation: a cardiovascular MR study. Int J Cardiol. 2016;223:639-44.

12. Connelly KA, Wijeysundera HC, Roifman I. Cardiac magnetic resonance imaging, Transcatheter aortic valve replacement, and early left ventricular mass regression-a virtual microscope into ventricular Remodelling? Can J Cardiol. 2016;32(12):1369-71.

13. Stroup DF, Berlin JA, Morton SC, Olkin I, Williamson GD, Rennie D, et al, Meta-analysis of observational studies in epidemiology: a proposal for reporting. Meta-analysis of observational studies in epidemiology (MOOSE) group. Jama. 2000;283(15):2008-12.

14. van Zuuren EJ, Fedorowicz Z. Moose on the loose: checklist for metaanalyses of observational studies. Br J Dermatol. 2016;175(5):853-4.

15. Dobson LE, Musa TA, Uddin A, Fairbairn TA, Swoboda PP, Erhayiem B, et al. Acute reverse Remodelling after Transcatheter aortic valve implantation: a link between myocardial fibrosis and left ventricular mass regression. Can J Cardiol. 2016:32(12):1411-8.

16. Stang A. Critical evaluation of the Newcastle-Ottawa scale for the assessment of the quality of nonrandomized studies in meta-analyses. Eur J Epidemiol. 2010;25(9):603-5.

17. Lo CK, Mertz D, Loeb M. Newcastle-Ottawa scale: comparing reviewers' to authors' assessments. BMC Med Res Methodol. 2014;14:45.

18. Riley RD, Higgins JP, Deeks JJ. Interpretation of random effects metaanalyses. BMJ (Clinical research ed). 2011;342:d549.

19. Higgins JP, Thompson SG, Deeks JJ, Altman DG. Measuring inconsistency in meta-analyses. BMJ (Clinical research ed). 2003;327(7414):557-60.

20. Egger M, Davey Smith G, Schneider M, Minder C. Bias in meta-analysis detected by a simple, graphical test. BMJ (Clinical research ed). 1997; 315(7109):629-34

21. Musa TA, Uddin A, Fairbairn TA, Dobson LE, Sourbron SP, Steadman CD, et al. Assessment of aortic stiffness by cardiovascular magnetic resonance 
following the treatment of severe aortic stenosis by TAVI and surgical AVR. J Cardiovasc Magn Reson. 2016;18(1):37.

22. Musa TA, Uddin A, Swoboda PP, Fairbairn TA, Dobson LE, Singh A, et al. Cardiovascular magnetic resonance evaluation of symptomatic severe aortic stenosis: association of circumferential myocardial strain and mortality. J Cardiovasc Magn Reson. 2017;19(1):13.

23. Dobson LE, Musa TA, Uddin A, Fairbairn TA, Blackman DJ, Ripley DP, et al. Gender influences left ventricular remodelling in the setting of aortic stenosis but does not appear to impact on reverse remodelling following transcatheter aortic valve implantation. J Cardiovasc Magn Reson. 2015; 17(Suppl 1):P332.

24. Uddin AU, Fairbairn TAF, Motwani MM, Kidambi AK, Steadman CS, Schlosshan DS, et al. 145 aortic regurgitation after COREVALVE TRANSCAT HETER aortic valve implantation (TAVI): assessment by echocardiography and cardiovascular magnetic resonance. Heart. 2013:99(suppl 2):A85-A6.

25. Gastl M, Behm P, Haberkorn S, Holzbach L, Veulemans V, Jacoby C, et al. Role of T2 mapping in left ventricular reverse remodeling after TAVR. Int J Cardiol. 2018;266:262-8.

26. Nucifora G, Tantiongco JP, Crouch G, Bennetts J, Sinhal A, Tully PJ, et al. Changes of left ventricular mechanics after trans-catheter aortic valve implantation and surgical aortic valve replacement for severe aortic stenosis: a tissue-tracking cardiac magnetic resonance study. Int I Cardiol. 2017:228:184-90.

27. Fairbairn TA, Steadman CD, Mather AN, Motwani M, Blackman DJ, Plein S, et al. Assessment of valve haemodynamics, reverse ventricular remodelling and myocardial fibrosis following transcatheter aortic valve implantation compared to surgical aortic valve replacement: a cardiovascular magnetic resonance study. Heart. 2013;99(16):1185-91.

28. La Manna A, Sanfilippo A, Capodanno D, Salemi A, Cadoni A, Cascone I, et al. Left ventricular reverse remodeling after transcatheter aortic valve implantation: a cardiovascular magnetic resonance study. J Cardiovasc Magn Reson. 2013;15:39.

29. Uddin A, Fairbairn T, Steadman CD, Herzog BA, Motwani M, Kidambi A, et al. Quantitative analysis of post-TAVI aortic regurgitation with cardiovascular magnetic resonance and the relationship to transthoracic echocardiography. J Cardiovasc Magn Reson. 2013;15(Suppl 1):114.

30. Gruenig S, Bönner F, Boering YC, Merx MW, Zeus T, Kelm M, et al. Left ventricular remodeling after transcatheter aortic valve implantation (TAVI). J Cardiovasc Magn Reson. 2013;15(Suppl 1):E39.

31. Richardson J, Cunnington M, Wong D, Bertaso A, Nelson A, Azarisman S, et al. Cardiac magnetic resonance imaging after Transcatheter aortic valve implantation. Heart Lung Circ. 2012;21:S188-S9.

32. Musa TA, Plein $S$, Greenwood JP. The role of cardiovascular magnetic resonance in the assessment of severe aortic stenosis and in postprocedural evaluation following transcatheter aortic valve implantation and surgical aortic valve replacement. Quant Imaging Med Surg. 2016; 6(3):259-73

33. St John Sutton M, Linde C, Gold MR, Abraham WT, Ghio S, Cerkvenik J, et al. Left ventricular architecture, long-term reverse remodeling, and clinical outcome in mild heart failure with cardiac resynchronization: results from the REVERSE trial. JACC Heart Fail. 2017:5(3):169-78.

34. Spinelli L, Morisco C, Assante di Panzillo E, Izzo R, Trimarco B. Reverse left ventricular remodeling after acute myocardial infarction: the prognostic impact of left ventricular global torsion. Int J C Imaging. 2013;29(4):787-95

35. Sato K, Kumar A, Jones BM, Mick SL, Krishnaswamy A, Grimm RA, et al. Reversibility of cardiac function predicts outcome after Transcatheter aortic valve replacement in patients with severe aortic stenosis. J Am Heart Assoc. 2017;6:7.

36. Ali A, Patel A, Ali Z, Abu-Omar Y, Saeed A, Athanasiou T, et al. Enhanced left ventricular mass regression after aortic valve replacement in patients with aortic stenosis is associated with improved long-term survival. J Thorac Cardiovasc Surg. 2011;142(2):285-91.

37. Hahn RT, Pibarot P, Stewart WJ, Weissman NJ, Gopalakrishnan D, Keane MG, et al. Comparison of transcatheter and surgical aortic valve replacement in severe aortic stenosis: a longitudinal study of echocardiography parameters in cohort a of the PARTNER trial (placement of aortic transcatheter valves). J Am Coll Cardiol. 2013;61(25):2514-21.

38. Rogers T, Waksman R. Role of CMR in TAVR. J Am Coll Cardiol Img. 2016; 9(5):593-602.
39. Fojt R, Motovska Z, Budera P, Maly M, Straka Z. Prognostic impact and change of concomitant mitral regurgitation after surgical or transcatheter aortic valve replacement for aortic stenosis. J Cardiol. 2016;67(6):526-30.

40. Poulin F, Yingchoncharoen T, Wilson WM, Horlick EM, Genereux P, Tuzcu $E M$, et al. Impact of prosthesis-patient mismatch on left ventricular myocardial mechanics after Transcatheter aortic valve replacement. J Am Heart Assoc. 2016;5:2.

41. Nazarian S, Hansford R, Rahsepar AA, Weltin V, McVeigh D, Gucuk Ipek E, et al. Safety of magnetic resonance imaging in patients with cardiac devices. N Engl J Med. 2017;377(26):2555-64.

\section{Publisher's Note}

Springer Nature remains neutral with regard to jurisdictional claims in published maps and institutional affiliations.
Ready to submit your research? Choose BMC and benefit from:

- fast, convenient online submission

- thorough peer review by experienced researchers in your field

- rapid publication on acceptance

- support for research data, including large and complex data types

- gold Open Access which fosters wider collaboration and increased citations

- maximum visibility for your research: over $100 \mathrm{M}$ website views per year

At BMC, research is always in progress.

Learn more biomedcentral.com/submissions 